J. Clin. Chem. Clin. Biochem.

Vol. 19, 1981, pp. 7-11

\title{
Enzymatische Bestimmung der Glycerinphosphatide im Serum unter Verwendung von Phospholipase $C$
}

\author{
Von K. J. Kornmüller \\ Aus dem Zentrallaboratorium (Chefarzt: Priv.-Doz. Dr. O. Müller-Plathe) \\ des Allgemeinen Krankenhauses Altona, Hamburg
}

(Eingegangen am 25. Februar 1980)

Zusammenfassung: Es wird ein einfaches Verfahren beschrieben, das die Bestimmung der Glycerinphosphatide im Serum mit Ausnahme der Lyso-Phosphatide erlaubt. Dazu sind zwei Glyceridbestimmungen erforderlich, eine aus Nativserum, entsprechend der üblichen „Triglycerid“-Bestimmung, die andere nach Inkubation des Serums mit Phospholipase C. Die Phospholipase C spaltet die Glycerinphosphatide in Cholinphosphat und Diglyceride, die zusätzlich zu Mono-, Di-, Triglyceriden und freiem Glycerin des Nativserums erfaßt werden. Die Differenz beider Glyceridbestimmungen entspricht der Glycerinphosphatidkonzentration. Die Bedeutung der vorliegenden Methode ergibt sich unter anderem aus der Beteiligung der Glycerinphosphatide an Gerinnungsvorgängen sowie deren Erhöhung bei gewissen Lebererkrankungen. Gegenüber der üblichen Bestimmung des Phospholipid-Phosphors bietet sie eine erhebliche Arbeitserleichterung und als vollenzymatische Methode eine höhere Spezifität.

\section{Enzymatic determination of serum glycerophosphatides, using phospholipase $C$}

Summary: A simple method is described for the determination of serum glycerophosphatides, with the exception of lyso-phosphatides. Two determinations are necessary. The usual "triglyceride" determination is performed on native serum, and a further determination is performed after incubation of the serum with phospholipase C. Glycerophosphatides are hydrolysed to diglycerides and phosphorylcholine by phospholipase $\mathrm{C}$. The resulting diglycerides are determined in addition to the mono-, di-, triglycerides and free glycerol of the native serum. The difference between the two glyceride determinations corresponds to the concentration of glycerophosphatides. Involvement of glycerophosphatides in blood coagulation and their increased levels in certain liver illnesses are two reasons for the importance of the present method. Compared with the usual method of measuring phospholipid phosphorus, the present method involves considerably less work, and, as a fully enzymatic method, it has a higher specificity.

\section{Einführung}

Cholesterin und Triglyceride sind heutzutage als Risikofaktoren der Arteriosklerose anerkannt und werden routinemäßig bestimmt. Die Phospholipide als weiterer Lipidbestandteil der Lipoproteine haben wegen der aufwendigen Analyseverfahren $(1,2)$ keinen Eingang in die Routinediagnostik gefunden. An den thrombotischen Komplikationen der Arteriosklerose sind die Phospholipide jedoch maßgeblich beteiligt. Sie sind für den Gerinnungseintritt sowohl über das Extrinsicals auch Intrinsic.-System erforderlich. Dabei kommt den Glycerinphosphatiden die entscheidende Bedeutung zu. Die thromboplastische Wirkung des Phosphatidylethanolamin ist unbestritten $(3,4,5)$. Eine Steigerung dieser Wirkung wird für die Kombination mit Phosphatidylserin (6) oder Phosphatidylcholin (7) beschrieben. Andere Autoren $(8,9)$ fanden die höchste thromboplasti- sche Aktivität bei der Kombination von Phosphatidylcholin mit Phosphatidylserin.

Im folgenden wird ein einfaches, auch routinemäßig anwendbares Verfahren beschrieben, das die Erfassung dieser Glycerinphosphatide im Serum mit Hilfe je einer Glyceridbestimmung vor und nach Inkubation mit Phospholipase $C$ erlaubt.

\section{Material und Methode}

Testprinzip (Abb. 1)

Die Glycerinphosphatide werden zunächst durch Inkubation des Serums mit Phospholipase $C$ in Cholinphosphat und Diglyceride gespalten. Eine anschließend durchgefürte „Glycerid"-Bestimmung erfaßt diese Diglyceride zusätzlich zu den Mono-, Di-, Triglyceriden und dem freien Glycerin im Nativserum. Die Differenz der ,Glycerid"-Konzentrationen nach Vorinkubation mit Phospholipase $C$ und im Nativserum ergibt die GlycerinphosphatidKonzentration. 


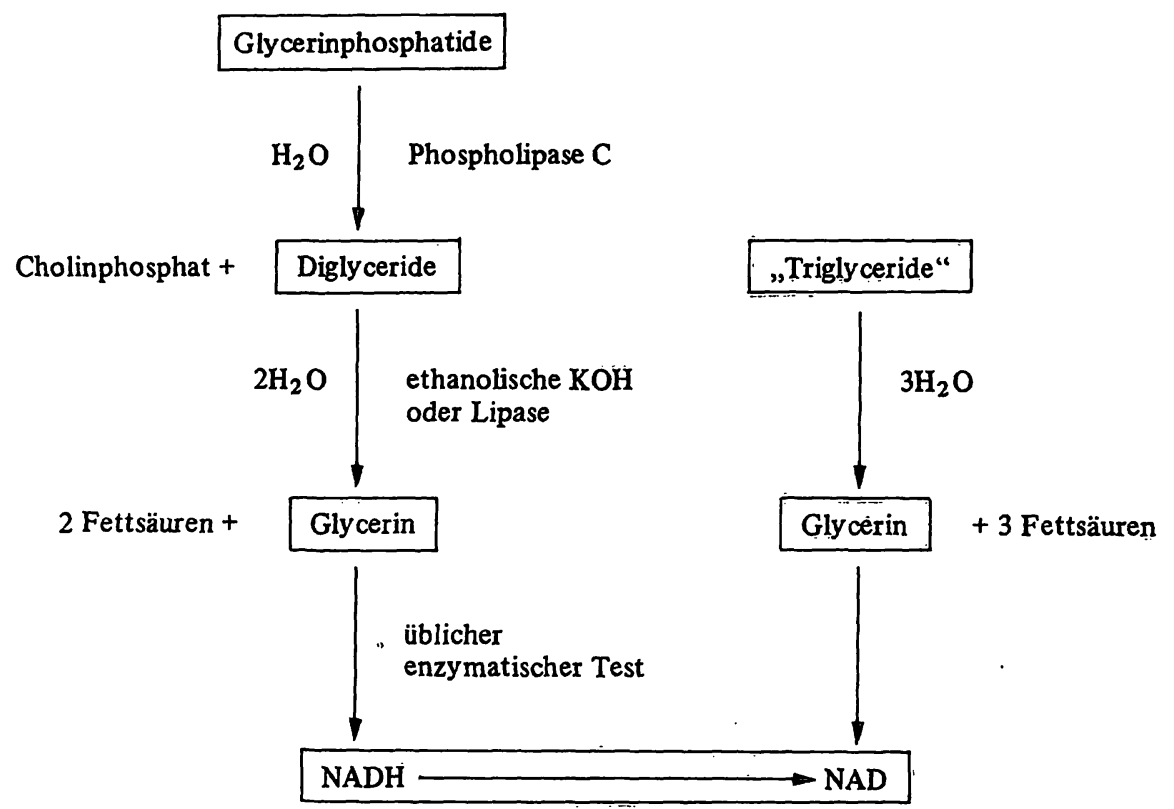

Abb. 1. Prinzip der Glycerinphosphatid-Bestimmung

Untersuchungsmaterial

Wiederfindungsuntersuchungen

a) Synthetische Phospholipide

$L-\alpha$-Lecithin (Dipalmitoyl), Calbiochem 4295

$L-\alpha$-Phosphatidylcholine (Dipalmitoyl), Sigma P 6769

$L-\alpha$-Phosphatidylcholine (Dimyristoyl), Sigma P 0880

$L-\alpha$-Phosphatidy lethanolamine (Dipalmitoyl), Sigma P 0890

Lösung in 1 Teil Triton $\mathrm{X}-100^{\circledR}+9$ Teile DiethylbarbituratPufferlösung pH 7,6

b) Lipostabil ${ }^{\circledR}$ Ampullen (Cholinphosphorsäuredigly ceridester $500 \mathrm{mg} / 10 \mathrm{ml}$ )

c) Kontrollseren mit einer Sollwertangabe für den PhospholipidPhosphor:

Seronorm-Lipid

Seronorm

Pathonorm H

Pathonorm L

Precinorm U

Validate LA

\section{Linearität}

a) Serum mit hoher Glycerinphosphatid-Konzentration

b) Lipostabil ${ }^{\circledR}$

Die Verdünnungen wurden mit Diethylbarbiturat-Acetat-Pufferlösung pH 7,6 (Behring ORHW 40/41) hergestellt.

\section{Ermittlung von Referenzwerten}

141 Seren von ambulanten Patienten und vom Personal mit normalen Lipidwerten (Cholesterin $<6,73 \mathrm{mmol} / \mathrm{l}$, Triglyceride $<2,28 \mathrm{mmol} / \mathrm{l}$ ) sowie unauffälligem Leberstatus (Bilirubin $<17,1 \mu \mathrm{mol} / 1$, alkalische Phosphatase $<180 \mathrm{U} / 1, \gamma$-Glutamyltransferase $<28 / 18 \mathrm{U} / 1$, Aspartataminotransferase $<18 / 15 \mathrm{U} / 1$, Alaninaminotransferase $<22 / 18 \mathrm{U} / 1$ ).

Geräte

Autoanalyzer II, Technicon

Fließschema: Methode MG 008 modifiziert, Technicon GmbH, Juni 1974.

\section{Reagenzien}

1. Automatenpackung Triglyceride (Neutralfett), UV-Test, Boehringer 124966.
2. 0,6 mol/1 ethanolische $\mathrm{KOH}$, Technicon TBV-0001-A.

3. Phospholipase $C$ aus Bacillus cereus, $1000 \mathrm{U} / \mathrm{ml}$, Boehringer 241709.

Gebrauchslösung: Verdünnung 1:51 in dest. Wasser.

Inkubation mit Phospholipase $\mathrm{C}$

$200 \mu \mathrm{l}$ Probe werden mit $50 \mu \mathrm{l}$ Phospholipase C-Gebrauchslösung bei Raumtemperatur für 1 Stunde inkubiert.

Kalibrierung

Precilip Charge 660 und 662 entsprechend dem deklarierten Trigly ceridwert.

Berechnung der Glycerinphosphatid-Konzentration

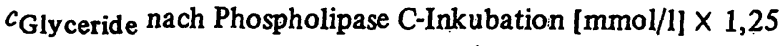

- $c_{\text {Gly ceride }}$ des Nativserums [mmol/1]

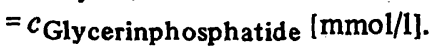

Der Faktor 1,25 ergibt sich aus der Verdünnung von $200 \mu 1 \mathrm{Se}$ rum mit $50 \mu 1$ Phospholipase C-Gebrauchslösung.

\section{Ergebnisse und Diskussion}

\section{Inkubationsbedingungen}

Ein pH-Optimum kann für die Phospholipase $\mathrm{C}$ nicht angegeben werden, da es von Substrat zu Substrat schwankt. Otnaess et al. (10) geben für Dipalmitylphos-, phatidylcholin einen optimalen Bereich von $\mathrm{pH} 8,0$ bis 8,3 an; für Phosphatidylethanolamin fanden sie (11) eine geringfügig höhere Hydrolyserate in Barbitalpuffer $\mathrm{pH} 7,4$ als bei $\mathrm{pH}$ 8,3. Eine Änderung des Substratspektrums der Phospholipase $C$ konnte zwischen pH 7,2 und 8,3 von diesen Autoren nicht festgestellt werden. Little (12) nennt als optimalen Bereich pH 7,5-8,0.

Der von uns gewählte Testansatz für die Bestimmung im Serum $(200 \mu \mathrm{l} \mathrm{Serum}+50 \mu \mathrm{l}$ der 1:51 mit dest. Wasser verdünnten Phospholipase-C-Lösung) ergab 2,5 und 
30 Minuten nach Inkubationsbeginn pH-Werte zwischen 7,9 und 7,6 und liegt damit in dem oben angegebenen Bereich. In ungepufferten Systemen wie beim Lipostabil oder den synthetischen Phospholipiden fällt der pH-Wert binnen 2,5 Minuten nach Phospholipase-C-Zugabe auf etwa 6,6 ab. Zum Lösen bzw. Verdünnen dieser Substanzen wurde daher ein Diethylbarbiturat-Acetat-Puffer $\mathrm{pH}$ 7,6 verwendet.

Als Inkubationstemperatur haben wir uns für Raumtemperatur und gegen $37^{\circ} \mathrm{C}$ entschieden. Eine höhere Inkubationstemperatur hätte zwar den Vorteil einer kürzeren Inkubationszeit, zuvor müßte allerdings der Einfluß von $37^{\circ} \mathrm{C}$ auf die Bestimmung der Glyceridkonzentration geprüft werden. Uns lag sehr an der Verwendbarkeit der im Rahmen der Glycerinphosphatidbestimmung ermittelten Triglyceridwerte für die Routinediagnostik.

Wie aus Abbildung 2 hervorgeht, sind die Serumansätze bei Raumtemperatur zumindest für 60 Minuten zu inkubieren. Manche Seren zeigen bereits nach 30 Minuten die maximale Glycerinphosphatid-Konzentration, wie das in Abbildung 2 für ein Kontrollserum dargestellt ist.

Für die Analyse synthetischer Glycerinphosphatide sind deutlich längere Inkubationszeiten erforderlich. Erst nach gut 3 Stunden werden hier die Maximalwerte erreicht.

Der Einfluß von Natriumdesoxycholat, das die Hydrolyse von gereinigtem Phosphatidylcholin mit langkettigen $\left(>C_{10}\right)$ Fettsäuren durch Phospholipase $\mathrm{C}$ beschleunigen soll (12), wurde mit Kontrollseren geprüft, die statt in dest. Wasser in $1 \mathrm{~g} / 1$ und $5 \mathrm{~g} / 1$ Desoxycholat gelöst wurden. Ein signifikanter Unterschied der gefundenen Glycerinphosphatid-Konzentrationen besteht nicht:

\section{$1 \mathrm{~g} / \mathrm{l}$ Desoxycholat: $\quad \overline{\mathrm{x}}=3,11 \mathrm{mmol} / \mathrm{l}$;}

$5 \mathrm{~g} / \mathrm{l}$ Desoxycholat: $\quad \overline{\mathrm{x}}=3,15 \mathrm{mmol} / \mathrm{l}$;

Desoxycholat-freier Ansatz: $\bar{x}=3,10 \mathrm{mmol} / 1$.

\section{Wiederfindung}

Bei der Analyse synthetischer Glycerinphosphatide, gelöst mit $100 \mathrm{~g} / 1$ Triton X-100 in DiethylbarbituratAcetat-Pufferlösung pH 7,6, können maximal 84-86\% wiedergefunden werden. Thre schlechte Angreifbarkeit durch Phospholipase $\mathrm{C}$ geht ja bereits aus Abbildung 2 hervor. Diese Befunde decken sich mit den Ergebnissen anderer Autoren $(11,13,14,15)$, die bei synthetischen Phospholipiden eine weniger vollständige Hydrolyse durch Phospholipase $\mathrm{C}$ als in biologischem Material fanden. Eine béssere Angreifbarkeit der Substrate scheint gegeben zu sein, wenn diese als Mizellen und nicht in monomolekularer-disperser Form vorliegen (12).

In Ermangelung eines Kontrollserums mit einer Sollwertangabe für Glycerinphosphatide wurde auf Lipostabil ${ }^{\circledR}$ zurückgegriffen. Dieses Medikament enthält Cholinphosphorsäurediglyceridester natürlicher Herkunft mit überwiegend ungesättigten Fettsäuren, davon $70 \%$ als Linolsäure, der Rest als Linolen- und Ölsäure. Die Glycerin-

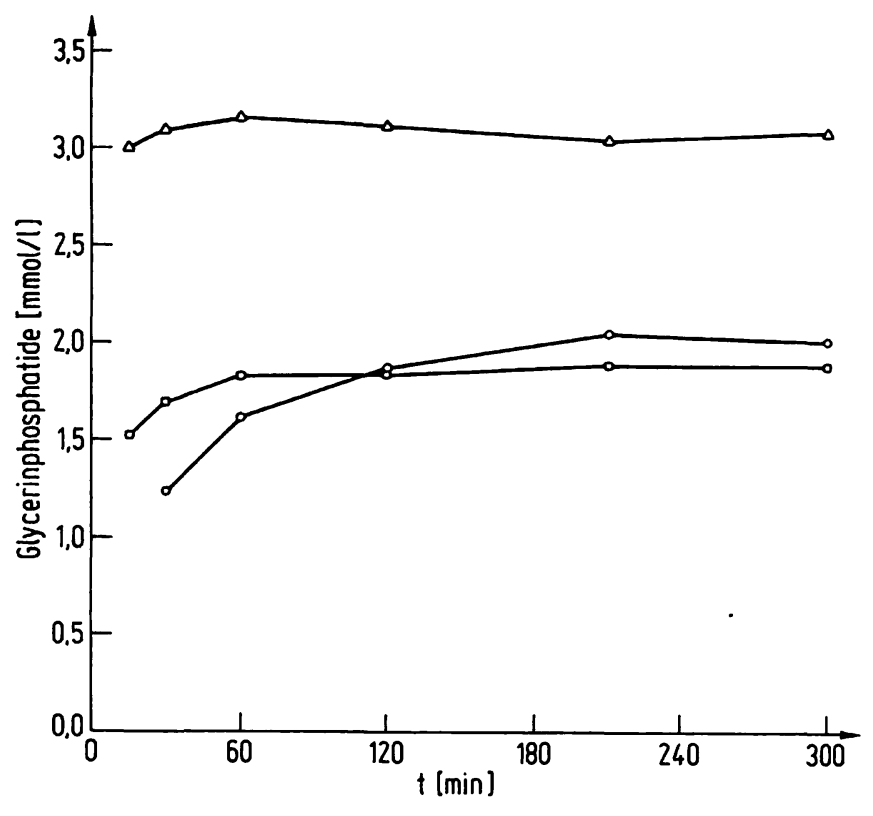

Abb. 2. Einfluß der Inkubationszeit bei Raumtemperatur auf die gemessene Glycerinphosphatid-Konzentration. 口- - Patientenserum

$\Delta-\Delta$ Kontrollserum (Seronorm Lipid)

○- o Synthetisches Phosphatidylcholin (Calbiochem) gelöst in $100 \mathrm{~g} / 1$ gepufferter Triton-Lösung.

phosphatid-Konzentration wird mit $5000 \mathrm{mg} / \mathrm{dl}$ angegeben, entsprechend $62,50 \mathrm{mmol} / 1$ bei einem berechneten Molekulargewicht von 800 .

Abbildung 3 gibt die Glycerinphosphatid-Konzentrationen verschiedener Lipostabil-Verdünnungen in Diethylbarbiturat-Acetat-Puffer wieder. Die Glycerinphosphatid-Konzentration im Lipostabil errechnet sich aufgrund der Regressionsgleichung mit 61,7 mmol/1. Das sind 98,7\% des Sollwertes.

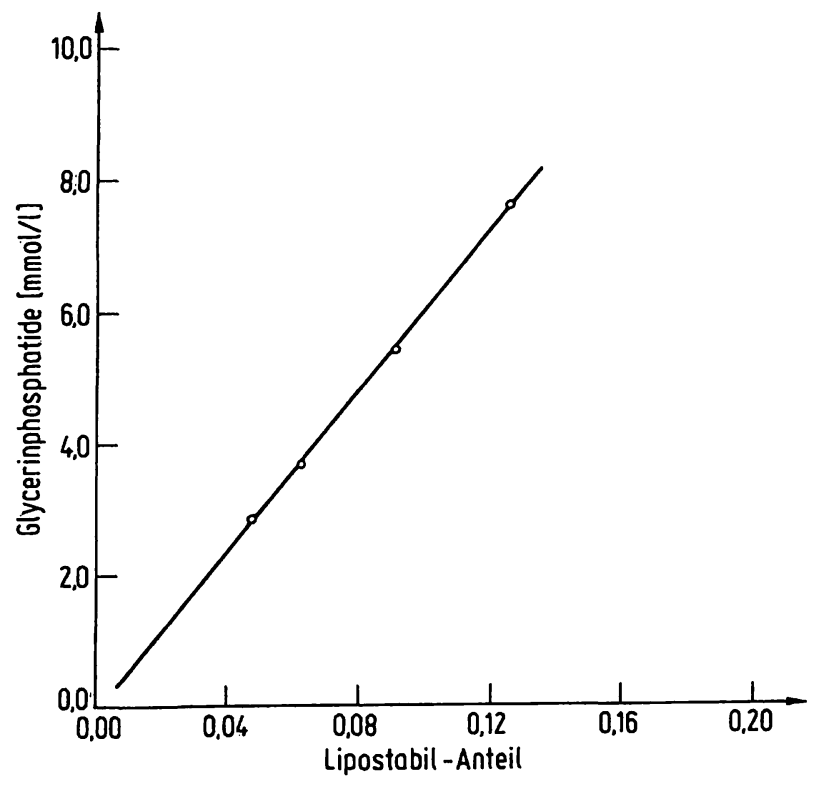

Abb. 3. Gly cerinphosphatid-Konzentrationen verschiedener Verdünnungen von Lipostabil. 
$\mathrm{Zu}$ fast den gleichen Glycerinphosphatid-Konzentrationen im Lipostabil gelangt man, wenn zu $200 \mu \mathrm{l}$ Serum 5 bzw. $20 \mu 1$ Lipostabil pipettiert werden. Als Mittelwert aus 8 Bestimmungen ergibt sich für die Zupipettierung von $5 \mu$ Lipostabil eine Erhöhung der GlycerinphosphatidKonzentration von $1,50 \mathrm{mmol} / \mathrm{l}$, bei $20 \mu \mathrm{l}$ von $5,48 \mathrm{mmol} / \mathrm{l}$. Unter Berücksichtigung des Verdünnungsfaktors errechnet sich die Glycerinphosphatidkonzentration im Lipostabil mit $61,5 \mathrm{mmol} / \mathrm{l}(98,4 \%)$ bzw. $60,3 \mathrm{mmol} / \mathrm{l}$ (96,5\% des Sollwertes).

Weiterhin wurden 6 Kontrollseren mit einer Sollwertangabe für den Phospholipid-Phosphor analysiert. Tabelle 1 gibt die gefundenen Glycerinphosphatid-Konzentrationen in Prozent der Sollwertangaben furr den Phospholipid-Phosphor wieder (rechte Spalte). Die Schwankungsbreite mit Werten zwischen 62,2 und $72,2 \%$ fällt relativ gering aus. Bedenkt man weiterhin, daß die Glycerinphosphatide etwa $70 \%$ der Phospholipide ausmachen, so sind die Werte durchaus plausibel.

\section{Linearität}

Wie bereits aus Abbildung 3 (Lipostabil-Verdünnungen) hervorgeht, ist die vorliegende Methode zumindest bis etwa $8,0 \mathrm{mmol} / 1$ Glycerinphosphatide linear $\left(R^{2}=0,9994\right)$. In Patientenseren sind von uns derartig hohe Glycerinphosphatid-Konzentrationen bisher nicht ermittelt worden. Abbildung 4 zeigt die Verdünnungsreihe eines Patientenserums mit hohen Glycerinphosphatidwerten. Auch hier finden sich streng lineare Verhältnisse $\left(R^{2}=0,9998\right)$.

\section{Präzisionskriterien}

Mit einem VK in der Serie von $1,1 \%$ und einem VK von Tag zu Tag von $3,8 \%$ sind die Präzisionskriterien der vorgeschlagenen Methode zur Bestimmung der Glycerinphosphatide als gut zu bezeichnen (s. Tab. 2).

Tab. 1. Glycerinphosphatid-Konzentration mehrerer Kontrollseren im Vergleich zur Sollwertangabe für den Phospholipid-Phosphor. Ein Istwert-Sollwert-Verhältnis von etwa $70 \%$ entspricht dem Anteil der Glycerinphosphatide an den Gesamt-Phospholipiden.

\begin{tabular}{lllc}
\hline Kontrollserum & $\begin{array}{l}\text { Phospho- } \\
\text { lipid- } \\
\text { Phosphor } \\
\text { Sollwert }\end{array}$ & Glycerinphosphatide \\
& Istwert & $\begin{array}{c}\text { Istwert/ } \\
\text { Sollwert }\end{array}$ \\
\hline Seronorm Lipid & 4,40 & 3,11 & 70,7 \\
Seronorm & 1,80 & 1,30 & 72,2 \\
Pathonorm H & 2,81 & 1,80 & 64,1 \\
Pathonorm L & 1,52 & 1,01 & 66,4 \\
Validate LA & 5,50 & 3,59 & 65,3 \\
Precinorm U & 2,30 & 1,43 & 62,2 \\
\hline
\end{tabular}

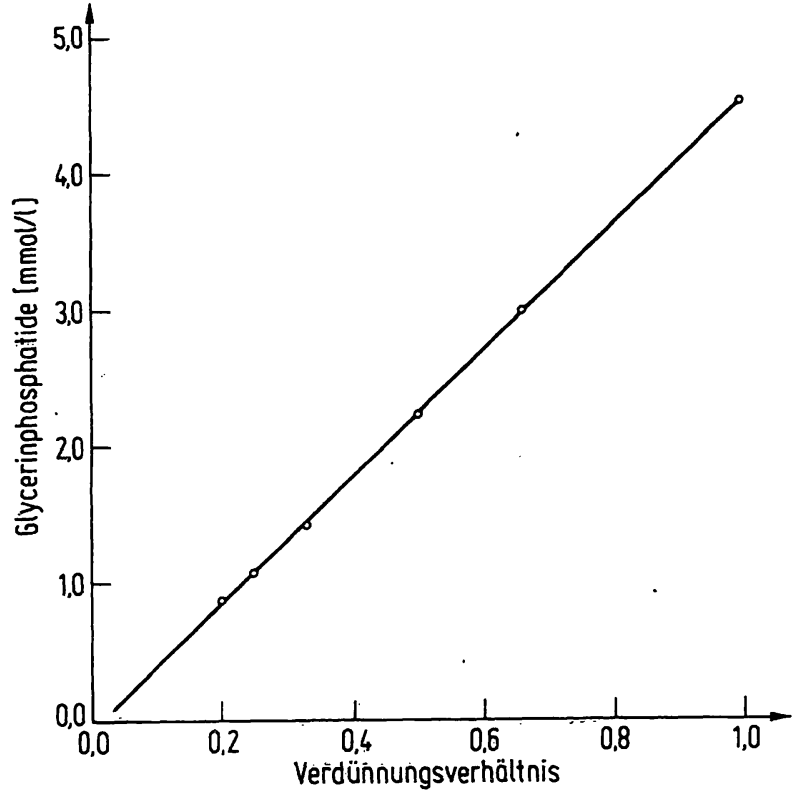

Abb. 4. Gly cerinphosphatid-Konzentrationen einer Serumverdünnungsreihe (Ausgangskonzentration: $4,56 \mathrm{mmol} / \mathrm{l}$ )

Tab. 2. Präzisionskriterien der vorgeschlagenen Glycerinphosphatid-Methode.

\begin{tabular}{lllll}
\hline & $\overline{\mathrm{x}}(\mathrm{mmol} / \mathrm{l})$ & $\mathrm{s}(\mathrm{mmol} / \mathrm{l})$ & $\mathrm{VK}(\%)$ & $\mathrm{n}$ \\
\hline $\begin{array}{l}\text { Präzision } \\
\text { in der Serie }\end{array}$ & 1,77 & 0,02 & 1,1 & 12 \\
$\begin{array}{l}\text { Präzision } \\
\text { von Tag zu Tag }\end{array}$ & 2,69 & 0,10 & 3,8 & 20 \\
\hline
\end{tabular}

\section{Ermittlung von Referenzwerten}

Abbildung 5 zeigt das Histogramm von 141 gesunden Erwachsenen. Der Referenzbereich erstreckt sich bei der Berechnung aus Mittelwert \pm 2 Standardabweichungen von 1,22 bis $2,42 \mathrm{mmol} / 1$, unter Berücksichtigung der 2,5- und 97,5-Percentilen von 1,28 bis $2,50 \mathrm{mmol} / 1$ Glycerinphosphatide. Diese Werte stimmen gut mit den Referenzwertangaben (16) für den Phospholipid-Phosphor überein: 1,94 bis $3,21 \mathrm{mmol} / 1$ bei einem Mittelwert von $2,58 \mathrm{mmol} / 1$. Unser Mittelwert von $1,82 \mathrm{mmol} / 1$ Glycerinphosphatide ergibt bei deren Anteil von $70 \%$ an den Gesamt-Phospholipiden (17) 2,60 mmol/1.

\section{Substratspezifität der Phospholipase C aus Bacillus cereus}

Systematische Untersuchungen wurden von uns zu diesem Thema nicht durchgeführt. Die Arbeitsgruppe um 


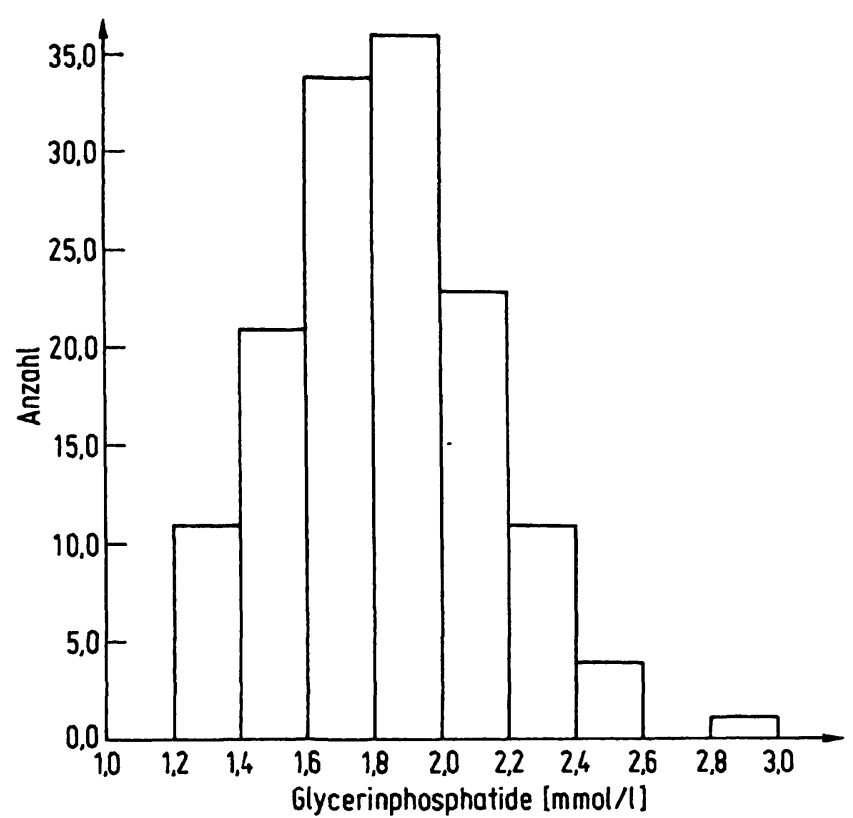

\section{Literatur}

1. Zilversmit, D. B. \& Davis, A. K. (1950), J. Lab. Clin. Med. $35,155-160$.

2. Wachter, H. (1965), Ärtl. Lab. 11, 11-15.

3. O'Brien, J. R. (1956), J. Clin. Pathol. 9, 47-51.

4. Rouser, G., White, S. G. \& Schloredt, D. (1958), Biochim. Biophys. Acta 28, 71-80.

5. Hoelzl-Wallach, D. F., Maurice, P. A., Steele, B. B. \& Surgenor, D. M. (1959), J. Biol. Chem. 234, 2829-2834.

6. Hecht, E. \& Slotta, K. H. (1962), Amer. J. Clin. Pathol. 37, 126-133.

7. Rapport, M. M. (1956), Nature 178, 591-592.

8. Therriault, D., Nichols, T. \& Jensen, H. (1958), J. Biol. Chem. 233, 1061-1065.

9. Troup, S. B., Reed, C. F., Marinetti, G. V. \& Swisher, S. N. (1960), J. Clin. Invest. 39, 342-351.

10. Otnaeșs, A.-B., Prydz, H., Bjфrklid, E. \& Berre, A. (1972), Eur. J. Biochem. 27, 238-243.
Otnaess, Little et al. $(11,12)$ hat sich eingehend mit der Substratspezifität beschäftigt. Danach werden durch Phospholipase $\mathrm{C}$ aus Bacillus cereus Phosphatidylcholin (Lecithin) sowie Phosphatidylethanolamin (Kephalin) und Phosphatidylserin (Kephalin) hydrolysiert, nicht jedoch die entsprechenden Lyso-Phosphatide. Sphingomyelin als zweitgrößte Phospholipidfraktion des Serums enthält kein Glycerin und wird zudem durch Phospholipase $\mathrm{C}$ aus Bacillus cereus nicht angegriffen.

Abb. 5. Häufigkeits-Verteilung der Glycerinphosphatidkonzentration im Serum bei 141 Erwachsenen mit normalem Leber- und Lipidstatus.

11. Otnaess, A.-B., Little, C., Sletten, K., Wallin, R., Johnsen, S., Flengsrud, R. \& Prydz, H. (1977), Eur. J. Biochem. 79, 459-468.

12. Little, C. (1977), Acta Chem. Scand. B 31 (1977), 267272.

13. Roelofsen, B., Zwaal, R. F. S., Comfurius, P., Woodward, C. B. \& van Deenen, L. L. M. (1971), Biochim. Biophys. Acta 241, 925-929.

14. Gazitt, Y., Ohad, J. \& Loyter, A. (1975), Biochim. Biophys. Acta 382, 65-72.

15. Bangham, A. D. \& Dawson, R. M. C. (1962), Biochim. Biophys. Acta 59, 103-115.

16. Zöllner, N. \& Eberhagen, D. (1965), Untersuchung und Bestimmung der Lipoide im Blut, Springer Verlag Berlin, Heidelberg, New York.

17. Wagener, H., Lang, D. \& Frosch, B. (1964), Z. Ges. Exp. Med. $138,425-434$. 
. 